\title{
Toward decision-based acceptance criteria for Bioanalytical Method Validation: a proposal for discussion from the European Bioanalysis Forum
}

\author{
Philip Timmerman*,1, Michaela Golob², Joanne Goodman³ ${ }^{3}$ Magnus Knutsson ${ }^{4}$, Robert \\ Nelson ${ }^{5}$, Marianne Scheel Fjording ${ }^{6}$ \& Steve White ${ }^{7}$ \\ ${ }^{1}$ European Bioanalysis Forum, Havenlaan 86c b204, 1000 Brussels, Belgium \\ ${ }^{2}$ Nuvisan, Grafing, Germany \\ ${ }^{3}$ Medlmmune, Cambridge, UK \\ ${ }^{4}$ Ferring, Copenhagen, Denmark \\ ${ }^{5}$ Novimmune, Geneva, Switzerland \\ ${ }^{6}$ Novo Nordisk, Måløv, Denmark \\ ${ }^{7}$ GlaxoSmithKline, Ware, UK \\ *Author for correspondence: Tel.: +32 4799101 32; chair@e-b-f.eu
}

First draft submitted: 16 April 2018; Accepted for publication: 16 May 2018; Published online: 21 August 2018

Validation of bioanalytical assays has been widely discussed over the last decades. Since the early 1990s, industry and regulators have been working on a consensus model building on the conclusions from the conference on Analytical Methods Validation: Bioavailability, Bioequivalence and Pharmacokinetic studies [1]. This first international conference was held in December 1990 in Crystal City, Arlington, USA and was sponsored by the American Association of Pharmaceutical Scientists (AAPS), US FDA, Federation Internationale Pharmaceutique, Health Protection Branch (Canada) and Association of Official Analytical Chemists. The conference has been referred to as the Crystal City I (CC-I) conference, and became the template for subsequent Crystal City (CC) conferences held between 2000 and 2015 [2-5]. All current regional or international regulations related to validation of bioanalytical assays and industry publications on the subject were based on the principles and practices discussed and agreed at the CC-I conference. Although, Health Canada formally referred to the CC-I conference report for bioequivalence studies in 1996 [6], it was only in 2001 that the FDA actually issued a formal guidance, which has been recently updated [7]. This 2001 guidance was based largely on the discussions from the CC-II meeting, which refined the conclusions from 1990 after a decade of experience with the CC-I principles. During that decade, important progress was made in various areas of analytical chemistry. Subsequent CC meetings and other international meetings hosted by, for example, the European Bioanalysis Forum (EBF) (together with European Federation for Pharmaceutical Sciences (EUFEPS) in 2010) or the Japan Bioanalysis Forum [8] to comment and provide input into upcoming regulations in Europe and Japan, brought together the industry experts and regulators to refine the practices or agree (but sometimes also disagree) to add or modify regulatory requirements based on observed flaws in methods or enhancements in technology. Some of the added requirements were inspired by industry sharing expertise at meetings or in peer-reviewed literature, others were requested by the regulators as part of their observations during regular audits and/or inspections at the time of filings.

\section{Globalization of the Bioanalytical Guidelines}

During the last decade, the regulated bioanalytical community truly globalized. We have also observed Bioanalytical Method Validation Guidelines emerging in many regions of the world. Most of these guidelines were based upon the aforementioned 2001 FDA guidance and included many of the conclusions from the different CC meetings. And although all guidelines were built on the same principles, some small differences from one guideline to another created a huge problem for the global bioanalytical community on how to comply with all requirements in an efficient way. 
Hence, since 2010, and aligned with guidelines being issued in regions outside of the USA, the industry intensified their desire on harmonization of bioanalytical guidance. At first, and as can be read from the Open Letter to the Regulators in 2011, the request for harmonization referred to harmonized interpretation of the guidance principles by the industry and the regulators [9]. Going forward, the industry united under the umbrella of the Global Bioanalysis Consortium, which in turn reported back on their discussion by issuing a number of high quality publications summarizing industry's current thinking on best practices for Bioanalytical Method Validation [10]. From there, industry's focus gradually shifted to a request for harmonization of the different regional guidelines and guidance involving the International Council for Harmonisation of Technical Requirements for Pharmaceuticals for Human Use (ICH), a proposal that was also made by the regulators, leading to the current development of the ICH M10 guideline [11].

\section{Adding more details in the guidelines}

As mentioned in the introduction of this manuscript, since the early 1990s of the 20th century, all discussions and practices built on the principles and paradigms of the CC-I paper, discussing the criteria for chemical assays on the one hand and ligand-binding assays (LBAs) on the other hand. Already in 2001, industry and regulators considered the progress in chemical assays and included more assay-specific requirements for the booming LC-MS area, dealing with matrix effects being the most prominent assay challenge. Also, the 2001 FDA guidance was far from complete or even satisfactory for the LBA community as exemplified by the CC-II follow-up meeting and subsequent meeting report on the best practices for these assay formats [12,13]. The DeSilva publication certainly added more details for LBA assays and became the foundation for LBA assay validations for the next decade [13].

\section{New modalities \& technologies}

Of course, from 2001 to date, technology progress did not stop and it will not stop tomorrow. Equally important, drug $R \& D$ portfolios continue to branch into many different areas and new modalities, requiring the bioanalytical scientist to reposition existing technology used in the laboratory or to integrate completely new analytical platforms. For one, this leads to a tremendous refinement of existing technology, both for chemical and LBAs with respect to the sensitivity, specificity and selectively. But also, it requires the bioanalytical scientists to combine assay formats or introduce new techniques into their (regulatory) bioanalytical toolbox.

In June 2011, the EBF hosted a Focus Meeting discussing the progress in technology allowing chemical assays to be used for a broader array of compounds historically only analyzed by binding assays [14]. One of the desires expressed at that meeting was not to simply 'copy/paste' acceptance technology-based acceptance criteria when analyzing peptides and proteins with MS-based assays, but to reflect if a cutting-edge protein analysis using LC-MS and the decisions taken with the data really need more stringent acceptance criteria than if the same molecule would have been analyzed using the conventional LBA. These were early days for peptide and protein analysis using LC-MS. During the years following the 2011 EBF Focus Meeting, industry experienced an exponential increase in technological opportunities to analyze larger molecules using MS detection and quantification. A steep learning curve, which is still continuing.

\section{Technology-based versus decision-based acceptance criteria}

In June 2017, the EBF hosted a second Focus Workshop on the theme in support of developing Bioanalytical Strategies for Large Molecules in Modern Drug Development [15]. In the meantime, many bioanalytical laboratories were developing and validating a broad array of assays for the quantification of peptides and (large) proteins using immunocapture and/or direct protein digest LC-MS(/MS) methods replacing or complementing the traditional LBA. Additionally, it has increasingly become unclear which acceptance criteria to apply, irrespective of a comprehensive AAPS paper published in 2015 [16]. During the workshop discussions, the idea developed that industry should take a step back and reflect on one of the starting principles of the Bioanalytical Method Validation: should technology drive the acceptance criteria of bioanalytical assays? During the panel discussions at the workshop, the delegates discussed if the current acceptance criteria for bioanalytical assays, and the difference when one technology is used versus another, should not be appraised to come to harmonized criteria, independent from the technology, but driven by the decision taken with the data, that is, a pharmacokinetic (PK) and/or toxicokinetic assessment. What would be the appropriate criteria be if they were not driven by what we can achieve, but by what is needed to take decisions in support of patient safety and efficacy of (new) drug products? 
Since the 1990 CC-I paper [1], the industry and regulators based the discussions on which acceptance criteria to apply on the technology used: chromatographic assays were held to accuracy/precision within 15\% (20\% at LLOQ) and binding assays at accuracy/precision within 20\% (25\% at LLOQ/ULOQ), irrespective of the decisions taken on the data. As part of these discussions, and not unimportant to mention, we may have lost the fact that prior to 2001 the agreed accuracy/precision was $\pm 20 \%$ and $\pm 25 \%$ for chromatographic/chemical assays and LBAs, respectively.

The proposal made at the 2016 EBF Focus Workshop was followed by a lively discussion on redefining acceptance criteria for Bioanalytical Method Validation and basing them on the decisions taken on the data. In this way, the proposal can contribute to the harmonization efforts in the bioanalytical community: defining one harmonized level of acceptance criteria for assays used to make PK and safety decisions. A number of arguments were brought forward in support of this proposal. First, it provides an answer to a discussion, which keeps the industry busy ever since CC-II. Shortly after that meeting in 2000, where industry and regulators confirmed that they were comfortable with \pm 20 or $25 \%$ for chemical/chromatographic and LBA assays, respectively, the decision was taken to change acceptance criteria from 20 to $15 \%$ and 25 to $20 \%$ for the chemical/chromatographic and LBA assays, respectively. However, these tighter criteria were still technology-based and did not consider that we actually make identical PK or safety claims on these chromatography or LBA data even though they inherently may differ in accuracy and precision. Also, the accuracy and precision criteria themselves may even be irrelevant in the context of the downstream decision made on these data. Hence, we often heard the question: why should the acceptance criteria be different just because a different analytical platform is utilized?

Second, harmonized decision-based acceptance criteria can provide an acceptable answer to one of the key questions from the EBF Workshop 'Which criteria to use in so-called 'hybrid assays' or immunocapture and/or protein digest LC-MS(/MS) methods?'. Are these 'hybrid assays' chromatographic assays or LBAs? And, even more challenging, why would scientists develop a cutting edge 'hybrid assay' if they will be held to more stringent chromatography acceptance criteria for data supporting exactly the same PK or safety claim as an LBA for that same compound?

A third and maybe most important argument is related to the fact that new technologies and new modalities will continue to enter our world. In the coming years, other technologies will be required to provide the $\mathrm{PK} /$ toxicokinetics (TK) answers for regulatory review and labeling. How will we be managing or regulating the use of these new technologies? As an example, which category should be utilized for flow cytometry, qPCR, positron emission tomography (PET) imaging or the variety of new MS-based applications? Which criteria should apply when these technologies are used to support PK/safety claims similar to conventional LC-MS/MS or LBAs?

As a bioanalytical community, we are aware of the game-changing impact of the proposal made in this manuscript and realize it requires intense discussions and reflections. This is also not a proposal to bring acceptance criteria for chromatography-based assays to $\pm 20 \%$ or for LBAs to $\pm 15 \%$. Instead, we are making a plea to define and agree on harmonized criteria, which can support the decision made on dosing, PK and safety from the bioanalytical data. As a consequence, this discussion needs active involvement and input from the end users of these concentration data. Indeed, as the proposal is about applying decision-based acceptance criteria instead of technology-based criteria, input from the stakeholders about making these decisions is crucial. We should not start from a blank page either; we have almost three decades of experience with the current level of acceptance criteria. At the same time we should not forget that from the inception of the 4-6-XX paradigm, experts were already challenging whether this is the best way to accept/reject bioanalytical data for PK [17].

The above proposal may also help progress the intense discussion on which criteria to use for biomarker assays. Since biomarker assays are pharmacodynamic assays, they should not automatically be held to PK acceptance criteria. It may help to develop pharmacodynamic acceptance criteria, which likely are individual per biomarker entity. We would like to refer to an earlier EBF recommendation paper to define acceptance criteria for biomarker assays on the decisions taken from the assay data [18].

\section{Conclusion \& future perspective}

With this manuscript, the EBF wants to propose an open discussion whether it makes sense to move away from technology-based acceptance criteria in favor of decision-based acceptance criteria. We hope the discussion can get sufficient air time in industry, project teams and at upcoming meetings, either bioanalytically focused or with all stakeholders. We believe the proposal can alleviate the current ambiguity and nonadded value discussion on defining 'hybrid assay criteria'. Once integrated in our industry, harmonized decision-based acceptance criteria for 
bioanalytical assays in support of PK/safety will create a transparent platform to accept new technologies in the toolbox of the regulated bioanalytical (BA) scientist. And last but not least, the proposal should be seen as refining the criteria for studies 'in scope' of the guidelines. As advocated during the AAPS/EBF/JBF sister meetings [19,20], criteria of studies 'out of scope' should not automatically be held to these criteria but should be driven by scientific rationale considering decisions taken from the assay data.

\section{Disclaimer}

The views and conclusion presented in this paper are those of the European Bioanalysis Forum and do not necessarily reflect the representative affiliation or company's position on the subject.

\section{Financial \& competing interests disclosure}

The authors have no relevant affiliations or financial involvement with any organization or entity with a financial interest in or financial conflict with the subject matter or materials discussed in the manuscript. This includes employment, consultancies, honoraria, stock ownership or options, expert testimony, grants or patents received or pending, or royalties.

No writing assistance was utilized in the production of this manuscript.

\section{References}

1. Shah VP, Midha KK, Dighe S et al. Analytical methods validation: bioavailability, bioequivalence and pharmacokinetic studies. Eur. J. Drug Metab. Pharmacokinet. 16(4), 249-255 (1991).

2. Shah VP, Midha KK, Findlay JW et al. Bioanalytical method validation - a revisit with a decade of progress. Pharm. Res. 17(12), 1551-1557 (2000).

3. Viswanathan CT, Bansal S, Booth B et al. Quantitative bioanalytical methods validation and implementation: best practices for chromatographic and ligand binding assays. Pharm. Res. 24(10), 1962-1973 (2007).

4. Fast DM, Kelley M, Viswanathan CT et al. Workshop report and follow-up - AAPS workshop on current topics in GLP bioanalysis: assay reproducibility for incurred samples - implications of Crystal City recommendations. AAPS J. 11(2), 238-241 (2009).

5. Booth B, Arnold ME, DeSilva B et al. Workshop report: Crystal City V-quantitative bioanalytical method validation and implementation: the 2013 revised FDA guidance. AAPS J. 17(2), 277-288 (2015).

6. Health Canada. Guidance for Industry: Conduct and Analysis of Bioavailability and Bioequivalence Studies - Part B: Oral Modified Release Formulations (1996). https://www.canada.ca/en/health-canada.html

7. US FDA. Guidance for Industry: Bioanalytical Method Validation (2018). https://www.fda.gov/downloads/drugs/guidances/ucm070107.Pdf

8. Japan Bioanalysis Forum. http://bioanalysisforum.jp/en

9. Timmerman P, Lowes S, Fast DM et al. Request for global harmonization of the guidance for bioanalytical method validation and sample analysis. Bioanalysis 2(4), 683 (2010).

10. Timmerman P, Arnold M, De Silva B et al. Introduction to the proposals from the Global Bioanalysis Consortium Harmonization Team. AAPS J. 16(6), 1159-1161 (2014).

11. ICH. Multidisciplinary guidelines. www.ich.org/products/guidelines/multidisciplinary/article/multidisciplinary-guidelines

12. Findlay JW, Smith WC, Lee JW et al. Validation of immunoassays for bioanalysis: a pharmaceutical industry perspective. J. Pharm. Biomed. Anal. 21(6), 1249-1273 (2000).

13. DeSilva B, Smith W, Weiner R et al. Recommendations for the bioanalytical method validation of ligand-binding assays to support pharmacokinetic assessments of macromolecules. Pharm. Res. 20(11), 1885-1900 (2003).

14. EBF meeting: large meets small. http://bru2011.europeanbioanalysisforum.eu/slides

15. EBF bioanalytical strategies for large molecules in modern drug development: LBA and LC-MS united. http://focus201706.europeanbioanalysisforum.eu/fw-slides/

16. Jenkins R, Duggan JX, Aubry AF et al. Recommendations for validation of LC-MS/MS bioanalytical methods for protein biotherapeutics. AAPS J. 17(1), 1-16 (2015).

17. Kringle RO. An assessment of the 4-6-20 rule for acceptance of analytical runs in bioavailability, bioequivalence, and pharmacokinetic studies. Pharm. Res. 11(4), 556-560 (1994).

18. Timmerman P, Herling C, Stoellner D et al. European Bioanalysis Forum recommendation on method establishment and bioanalysis of biomarkers in support of drug development. Bioanalysis 4(15), 1883-1894 (2012).

19. Industry input into ICH M10: experimental data as the cornerstone for a science driven bioanalytical guideline. http://focus201709.europeanbioanalysisforum.eu/slides/ 
20. Achieving a Harmonized Guideline for Bioanalytical Method Validation: ICH M10. AAPS News Magazine. https://www.aapsnewsmagazine.org/aapsnewsmagazine/articles/2018/may18/cover-story-may18 
